\title{
ON THE SUMMABILITY OF FOURIER SERIES $\dagger$
}

\author{
BY \\ W. C. RANDELS
}

1. Notation. We shall use the following notation which is similar to that of Hille and Tamarkin $\ddagger$ [2]. We shall use $f(x)$ to denote a function periodic of period $2 \pi$ and integrable over $(-\pi, \pi)$. The Fourier series of $f(x)$ is

$$
\sum_{n=-\infty}^{\infty} f_{n} e^{i n x}
$$

where

$$
f_{n}=\frac{1}{2 \pi} \int_{-\pi}^{\pi} f(x) e^{-i n x} d x .
$$

The class of such functions and their related Fourier series is denoted by $L$. We define

$$
\tilde{f}(x)=\lim _{\epsilon \rightarrow 0}\left[-\frac{1}{2 \pi} \int_{\epsilon}^{\pi}\{f(x+t)-f(x-t)\} \cot \frac{t}{2} d t\right]
$$

and associate with it the series

$$
-i \sum_{n=-\infty}^{\infty} \operatorname{sgn} n f_{n} e^{i n x}
$$

We call the class of such series and their related functions $\tilde{L}$. If $f(x) \subset B . V$. on $(-\pi, \pi)$, we denote by $L^{\prime}$ the class of functions $f^{\prime}(x)$ and their related series, $\S$

$$
\frac{1}{2 \pi} \int_{-\pi}^{\pi} d_{t} f(x+t)+i \sum_{n=-\infty}^{\infty} n f_{n} e^{i n x}
$$

and by $\tilde{L}^{\prime}$ the class of functions

$$
f^{\prime}(x)=\lim _{\epsilon \rightarrow 0}\left[-\frac{1}{2 \pi} \int_{\epsilon}^{\pi} d_{t}\{f(x+t)+f(x-t)-2 f(x)\} \cot \frac{t}{2}\right]
$$

and their related series

$\dagger$ Presented to the Society, January 2, 1936; received by the editors March 7, 1936.

$\ddagger$ The numbers in brackets refer to the references at the end of the paper.

$\$$ The Stieltjes integral used in this paper is the Young-Stieltjes integral over the open interval so that $\int_{-\pi}^{\pi} d_{t} f(x+t)$ need not be zero. This usage differs from that of Hille and Tamarkin [2]. 


$$
\sum_{n=-\infty}^{\infty}|n| f_{n} e^{i n x}
$$

We shall use the symbol $\mathfrak{T}$ for any one of these four classes. A function of class $\mathfrak{I}$ will be denoted by $F(x)$ and the general term of the associated series by $A_{n}(x)$.

We define

$$
\begin{aligned}
\phi(L, t) & =f(x+t)+f(x-t)-2 f(x), \\
\phi(\tilde{L}, t) & =f(x+t)-f(x-t), \\
\phi_{0}(L, t) & =\int_{0}^{t} \phi(L, \tau) d \tau, \\
\phi_{0}(\tilde{L}, t) & =\int_{0}^{t} \phi(\tilde{L}, \tau) d \tau, \\
\phi_{0}\left(L^{\prime}, t\right) & =f(x+t)-f(x-t)-2 t f^{\prime}(x), \\
\phi_{0}\left(\tilde{L}^{\prime}, t\right) & =\phi(L, t) .
\end{aligned}
$$

We let $E(F, f)(E(\tilde{F}, f))$ be the set of points $x$ where $f(x)(\tilde{f}(x))$ has a definite value and $\phi(L, t) \rightarrow 0$ as $t \rightarrow 0(\phi(\tilde{L}, t) \rightarrow 0$ as $t \rightarrow 0)$ : If $\phi_{0}(\mathfrak{T}, t) \subset B . V$. on $(-\pi, \pi)$, we define $E(\mathfrak{T}, f)$ as the set of points where $F(x)$ has a definite value and $\dagger$

$$
\int_{0}^{t}\left|d \phi_{0}(\mathfrak{T}, \tau)\right|=o(t) \text { as } t \rightarrow 0 .
$$

We now consider the transformation of a series defined by means of a matrix $a_{m n}(\dot{m}, n=0,1, \cdots)$ in the following manner

$$
T_{m}(\mathfrak{T}, x) \sim \sum_{n=-\infty}^{\infty} a_{m|n|} A_{n}(x) .
$$

We make the restriction on $a_{m n}$ that it define a regular method of summability, that is:

$$
\sum_{n=0}^{\infty}\left|a_{m n}-a_{m, n+1}\right|<A, \quad A \text { not depending on } m,
$$

$$
a_{m n} \rightarrow 1 \text { as } m \rightarrow \infty \text {, for every } n
$$

and that the series

$$
\sum_{n=-\infty}^{\infty} a_{m|n|} A_{n}(x)
$$

$\dagger \mathrm{By} \int_{a}^{b}|d \phi(t)|$ we mean the total variation of $\phi(t)$ on the interval $(a, b)$. 
be a Fourier series (cf. Hille and Tamarkin [3]). Then $T_{m}(\mathfrak{T}, x)$ is considered as a function of class $L$ associated with (1.11). Thus $T_{m}(\mathfrak{T}, x)$ may be defined even though (1.11) does not converge. We denote the method of summability determined by the matrix $a_{m n}$ by $\mathscr{A}$.

If $E_{F}$ stands for a set of points at which $F(x)$ has a definite value, we can make the following definitions:

Definition 1. A method of summation $\mathfrak{A}$ is said to be ( $\left.\mathfrak{T}, E_{F}\right)$-effective if, whenever $F(x) \subset \mathfrak{T}$ and $x \subset E_{F}$,

$$
T_{m}(\mathfrak{I}, x)-F(x) \rightarrow 0 \text { as } m \rightarrow \infty .
$$

Definition 2. A method $\mathfrak{A}$ which is $\left(\mathfrak{T}, E_{P}\right)$-effective is said to be:

(i) $\quad F$-effective, if $\mathfrak{T}=L, E_{F}=E(F, f)$,

(ii) $\tilde{F}$-effective, if $\mathfrak{I}=\tilde{L}, E_{F}=E(\tilde{F}, f)$,

(iii) $L$-effective, if $\mathfrak{T}=L, E_{F}=E(L, f)$,

(iv) $\tilde{L}$-effective, if $\mathfrak{T}=\tilde{L}, E_{F}=E(\tilde{L}, f)$,

(v) $L^{\prime}$-effective, if $\mathfrak{T}=L^{\prime}, E_{F}=E\left(L^{\prime}, f\right)$,

(vi) $\tilde{L}^{\prime}$-effective, if $\mathfrak{T}=\tilde{L}^{\prime}, E_{F}=E\left(\tilde{L}^{\prime}, f\right)$.

2. $\mathfrak{T}=L$. By a theorem of Fekete [1] the most general factor sequence $\left\{a_{n}\right\}$ carrying a Fourier series into a Fourier series occurs when

$$
\sum_{n=1}^{\infty} \frac{a_{n}}{n} \sin n x \in B . V . \text { on }(-\pi, \pi),
$$

and

$$
\sum_{n=1}^{\infty} a_{n}\left\{f_{n} e^{i n x}+f_{-n} e^{-i n x}\right\} \sim \frac{1}{\pi} \int_{-\pi}^{\pi} f(x+t) d\left\{\sum_{n=1}^{\infty} \frac{a_{n}}{n} \sin n t\right\} .
$$

Then, if we let $\lim _{n \rightarrow \infty} a_{m n}=a_{m}$,

$$
T_{m}(L, x)=a_{m 0} f_{0}+\frac{1}{\pi} \int_{-\pi}^{\pi} f(x+t) d\left\{\sum_{n=1}^{\infty} \frac{a_{m}}{n} \sin n t+\sum_{n=1}^{\infty} \frac{a_{m n}-a_{m}}{n} \sin n t\right\} .
$$

But

$$
\sum_{n=1}^{\infty} \frac{\sin n x}{n}=\left\{\begin{array}{cc}
\frac{\pi-x}{2}, & 0<x<\pi, \\
\frac{-x-\pi}{2}, & -\pi<x<0, \\
0, & x=0,
\end{array}\right.
$$

so that 


$$
\frac{1}{\pi} \int_{-\pi}^{\pi} f(x+t) d\left\{\sum_{n=1}^{\infty} \frac{a_{m}}{n} \sin n t\right\}=a_{m} f(x)-a_{m} f_{0} .
$$

Therefore for our problem we need only consider

$$
\left(a_{m 0}-a_{m}\right) f_{0}+\frac{1}{\pi} \int_{-\pi}^{\pi} f(x+t) d\left\{\sum_{n=1}^{\infty} \frac{a_{m n}-a_{m}}{n} \sin n t\right\} .
$$

Hence it involves no loss of generality to suppose that $a_{m n} \rightarrow 0$ as $n \rightarrow \infty$, and we shall do so from now on.

Using Abel's method of partial summation we get

$$
\begin{aligned}
\sum_{n=1}^{\infty} a_{m n} \cos n x & =\sum_{n=1}^{\infty} a_{m n}\left\{S_{n}(x)-S_{n-1}(x)\right\} \\
& =-a_{m 1} S_{0}(x)+\sum_{n=1}^{\infty}\left\{a_{m n}-a_{m, n+1}\right\} S_{n}(x),
\end{aligned}
$$

where

$$
S_{n}(x)=\frac{1}{2}+\sum_{\nu=1}^{n} \cos \nu x=\frac{\sin \left(n+\frac{1}{2}\right) x}{2 \sin \frac{1}{2} x}
$$

Hence, by (1.9),

$$
\sum_{n=1}^{\infty} \frac{a_{m n}}{n} \sin n x
$$

is absolutely continuous on every interval not containing the origin. Moreover, again by Abel's transformation,

$$
\sum_{n=1}^{\infty} \frac{a_{m n}}{n} \sin n x=\sum_{n=1}^{\infty}\left(a_{m n}-a_{m, n+1}\right)\left(\sum_{\nu=1}^{n} \frac{1}{\nu} \sin \nu x\right)
$$

and, since the partial sums

$$
\sum_{\nu=1}^{n} \frac{1}{\nu} \sin \nu x
$$

are bounded, the function

$$
\sum_{n=1}^{\infty} \frac{a_{m n}}{n} \sin n x
$$

is continuous at the origin and so, since it is of bounded variation by hypothesis, it must be absolutely continuous on the interval $(-\pi, \pi)$. Hence we can write 


$$
\begin{aligned}
T_{m}(L, x) & =a_{m 0} f_{0}+\frac{1}{\pi} \int_{-\pi}^{\pi} f(x+t)\left\{-\frac{a_{m 1}}{2}+\sum_{n=1}^{\infty}\left(a_{m n}-a_{m, n+1}\right) \frac{\sin \left(n+\frac{1}{2}\right) t}{2 \sin \frac{1}{2} t}\right\} d t \\
& =\frac{1}{\pi} \int_{-\pi}^{\pi} f(x+t)\left\{\frac{a_{m 0}}{2}-\frac{a_{m 1}}{2}+\sum_{n=1}^{\infty}\left(a_{m n}-a_{m, n+1}\right) \frac{\sin \left(n+\frac{1}{2}\right) t}{2 \sin \frac{1}{2} t}\right\} d t \\
& =\frac{1}{\pi} \int_{-\pi}^{\pi} f(x+t)\left\{\sum_{n=0}^{\infty}\left(a_{m n}-a_{m, n+1}\right) \frac{\sin \left(n+\frac{1}{2}\right) t}{2 \sin \frac{1}{2} t}\right\} d t
\end{aligned}
$$

and

$$
T_{m}(L, x)-a_{m 0} f(x)=\frac{1}{\pi} \int_{0}^{\pi} \phi(L, t)\left\{\sum_{n=0}^{\infty}\left(a_{m n}-a_{m, n+1}\right) \frac{\sin \left(n+\frac{1}{2}\right) t}{2 \sin \frac{1}{2} t}\right\} d t .
$$

By the Riemann-Lebesgue theorem

$$
\int_{0}^{\pi} \phi(L, t)\left\{\frac{\sin \left(n+\frac{1}{2}\right) t}{2 \sin \frac{1}{2} t}-\frac{\sin n t}{t}\right\} d t=o(1) \text { as } n \rightarrow \infty
$$

so that, by the regularity of $\mathfrak{A}$,

$$
T_{m}(L, x)-f(x)=\frac{1}{\pi} \int_{0}^{\pi} \phi(L, t)\left\{\sum_{n=0}^{\infty} a_{m n}-a_{m, n+1} \frac{\sin n t}{t}\right\} d t+o(1) \text { as } m \rightarrow \infty .
$$

For convenience we define

$$
T_{m}=\frac{1}{\pi} \int_{0}^{\pi} \phi(L, t) \frac{1}{t} \sum_{n=0}^{\infty}\left(a_{m n}-a_{m, n+1}\right) \sin n t d t .
$$

We also define

$$
q_{m}(u)=\sum_{0 \leqq v<m u}\left(a_{m \nu}-a_{m, v+1}\right) .
$$

Then (1.9) can be written

$$
\int_{0}^{\infty}\left|d q_{m}(u)\right|<A .
$$

Then

$$
T_{m}=\frac{1}{\pi} \int_{0}^{\pi} \phi(L, t) \frac{1}{t} H_{m}(m t) d t,
$$

where

$$
H_{m}(t)=\int_{0}^{\infty} \sin u t d q_{m}(u) .
$$


In this notation the familiar necessary and sufficient condition for $F$-effectiveness is that there exist an $M$ such that

$$
\int_{0}^{\pi}\left|\frac{1}{t} H_{m}(m t)\right| d t<M .
$$

We wish to find a condition which will play a similar role for $L$-effectiveness. We introduce

$$
H_{m}=\sum_{n=0}^{\left[\log _{2} m\right]} \max _{2^{n} \leqq t \leqq 2^{n+1}}\left|H_{m}(t)\right| .
$$

We shall consider only methods of summation for which there exists a constant $B$ such that

$$
\left|H_{m}(t)\right|<B t \text {. }
$$

This condition is always satisfied for methods defined by trapezoidal matrices since for such methods there is an $N$ such that $q_{m}(u)=a_{m 0}$ for $u>N$, and hence

$$
\left|H_{m}(t)\right|=\left|\int_{0}^{\infty} \sin u t d q_{m}(u)\right|=\left|t \int_{0}^{\infty}\left[-a_{m 0}+q(u)\right] \cos u t d u\right|<A N t .
$$

We now propose to show that if (2.2) is satisfied

$$
T_{m}=o\left(H_{m}\right)+o(1)
$$

and furthermore that this result is the best possible in the sense that, if $\bar{H}_{m}=\max _{\mu \preceq m} H_{\mu} \rightarrow \infty$ as $m \rightarrow \infty$, then for every sequence $\left\{d_{m}\right\}$ such that $d_{m} \downarrow 0$ as $m \rightarrow \infty$ it is possible to find a function $f(x)$ and a point $x \subset E(L, f)$ such that

$$
T_{m} \neq O\left(d_{m} \bar{H}_{m}\right) \text {. }
$$

This implies that a necessary and sufficient condition for $L$-effectiveness of methods of summability satisfying (2.2) is the existence of an $M$ such that

$$
H_{m}<M \text {. }
$$

In order to prove (2.3) we notice that by the Riemann-Lebesgue theorem for every $a$

$$
\int_{a}^{\pi} \phi(L, t) \frac{\sin n t}{t} d t=o(1) \text { as } n \rightarrow \infty,
$$

so that by the regularity of $\mathfrak{A}$

$$
\int_{a}^{\pi} \phi(L, t) \frac{1}{t} H_{m}(m t) d t=o(1) \text { as } m \rightarrow \infty .
$$

Moreover, if $x \subset E(L, f)$, 


$$
\begin{aligned}
& \mid \int_{0}^{a} \phi(L, t) \frac{1}{t} H_{m}(m t) d t \mid \\
& \leqq \\
& \quad\left|\int_{0}^{1 / m} \phi(L, t) \frac{1}{t} H_{m}(m t) d t\right|+\left|\int_{1 / m}^{a} \phi(L, t) \frac{1}{t} H_{m}(m t) d t\right| \\
& \quad<B m \int_{0}^{1 / m}|\phi(L, t)| d t+\left|\int_{1}^{m a} \phi(L, t / m) \frac{1}{t} H_{m}(t) d t\right| \\
& \quad=o(1)+o\left(\sum_{n=0}^{\left[\log _{2} m a\right]} \max _{2^{n} \leqq t \leqq 2^{n+1}}\left|H_{m}(t)\right| 2^{-n} \int_{1}^{2^{n+1}}|\phi(L, t / m)| d t\right) \\
& \quad=o(1)+o\left(H_{m}\right) \text { uniformly in } m \text { as } a \rightarrow 0 .
\end{aligned}
$$

Then (2.3) follows from (2.6) and (2.7) by a familiar technique.

In order to show that this result is the best possible it is sufficient to prove that for every fixed $a$ it is possible to find a function $f(x)$ and an integer $m$ such that

$$
\frac{1}{\pi} \int_{1 / m}^{a} f(t) \frac{1}{t} H_{m}(m t) d t>\bar{H}_{m}
$$

and there is a constant $K$ independent of $a$ such that

$$
\int_{1 / m}^{t}|f(t)| d t<K t, \quad t \leqq a .
$$

For, if $d_{m} \downarrow 0$ is given, then making use of the Riemann-Lebesgue theorem and the regularity of $\mathfrak{A}$ we construct by induction a sequence of integers $\left\{m_{i}\right\}$ and functions $f_{i}(x)$ such that $m_{0}=1$, and

$$
\begin{gathered}
\sum_{i=1}^{\infty}\left(d_{m_{i}}\right)^{1 / 2}<\infty, \\
\left|\frac{1}{\pi} \int_{1 / m_{j}}^{1 / m_{j-1}} f_{j}(t) \frac{1}{t} H_{m_{i}}\left(m_{i} t\right) d t\right|<1, \quad j<i, \\
\frac{1}{\pi} \int_{1 / m_{i}}^{1 / m_{i-1}} f_{i}(t) \frac{1}{t} H_{m_{i}}\left(m_{i} t\right) d t>\bar{H}_{m_{i}},
\end{gathered}
$$

and

$$
\frac{1}{\pi} \int_{1 / m_{i}}^{t}\left|f_{i}(t)\right| d t<K t, \quad t \leqq \frac{1}{m_{i-1}}
$$

We define 


$$
f(x)=\left\{\begin{array}{cc}
\left(d_{m_{i}}\right)^{1 / 2} f_{i}(|x|), & \frac{1}{m_{i}} \leqq|x|<\frac{1}{m_{i-1}}, \\
0, \quad x=0, & 1 \leqq|x| \leqq \pi .
\end{array}\right.
$$

Then at $x=0, \phi(L, t)=2 f(t)$ and, by $(2.13)$, if $1 / m_{i} \leqq t \leqq 1 / m_{i-1}$,

$$
\begin{aligned}
\int_{0}^{t}|\phi(L, t)| d t & =2 \sum_{j=i+1}^{\infty}\left(d_{m_{j}}\right)^{1 / 2} \int_{1 / m_{j}}^{1 / m_{j-1}}\left|f_{j}(t)\right| d t+2\left(d_{m_{i}}\right)^{1 / 2} \int_{1 / m_{i}}^{t}\left|f_{i}(t)\right| d t \\
& <2 K t \sum_{j=i}^{\infty}\left(d_{m_{j}}\right)^{1 / 2}=o(t) \text { as } t \rightarrow 0
\end{aligned}
$$

so that the point $x=0$ is in $E(L, f)$. Moreover

$$
\begin{aligned}
T_{m_{i}}= & \frac{1}{\pi} \int_{0}^{\pi} \phi(L, t) \frac{1}{t} H_{m_{i}}\left(m_{i} t\right) d t \\
= & \frac{1}{\pi} \int_{0}^{1 / m_{i}} \phi(L, t) \frac{1}{t} H_{m_{i}}\left(m_{i} t\right) d t+\frac{1}{\pi} \int_{1 / m_{i}}^{1 / m_{i-1}} \phi(L, t) \frac{1}{t} H_{m_{i}}\left(m_{i} t\right) d t \\
& +\frac{1}{\pi} \int_{1 / m_{i-1}}^{\pi} \phi(L, t) \frac{1}{t} H_{m_{i}}\left(m_{i} t\right) d t .
\end{aligned}
$$

By (2.2)

$$
\left|\frac{1}{\pi} \int_{0}^{1 / m_{i}} \phi(L, t) \frac{1}{t} H_{m_{i}}\left(m_{i} t\right) d t\right|<\frac{B m_{i}}{\pi} \int_{0}^{1 / m_{i}}|\phi(L, t)| d t=o(1)
$$

and by (2.11)

$$
\begin{aligned}
\frac{1}{\pi} \int_{1 / m_{i-1}}^{\pi} \phi(L, t) \frac{1}{t} H_{m_{i}}\left(m_{i} t\right) d t & =\sum_{j=1}^{i-1} \frac{2\left(d_{m_{j}}\right)^{1 / 2}}{\pi} \int_{1 / m_{j}}^{1 / m_{j-1}} f_{j}(t) \frac{1}{t} H_{m_{i}}\left(m_{i} t\right) d t \\
& =\dot{O}\left(\sum_{j=1}^{\infty}\left(d_{m_{j}}\right)^{1 / 2}\right)=O(1) .
\end{aligned}
$$

Finally by (2.12)

$$
\begin{aligned}
\frac{1}{\pi} \int_{1 / m_{i}}^{1 / m_{i}-1} \phi(L, t) \frac{1}{t} & H_{m_{i}}\left(m_{i} t\right) d t \\
& =\frac{2\left(d_{m_{i}}\right)^{1 / 2}}{\pi} \int_{1 / m_{i}}^{1 / m_{i-1}} f(t) \frac{1}{t} H_{m_{i}}\left(m_{i} t\right) d t>2\left(d_{m_{i}}\right)^{1 / 2} \bar{H}_{m_{i}},
\end{aligned}
$$

so that

$$
T_{m_{i}}>2\left(d_{m_{i}}\right)^{1 / 2} \bar{H}_{m_{i}}-o(1)-O(1) \neq \neq\left(d_{m_{i}} \bar{H}_{m_{i}}\right)
$$


It only remains now to prove the existence of an $f(x)$ and an $m$ with the properties $(2.8),(2.9)$. We notice that

$$
\left|H_{m}(t)\right|=\left|\int_{0}^{\infty} \sin u t d q_{m}(u)\right|<\int_{0}^{\infty}\left|d q_{m}(u)\right|<A,
$$

so that for a fixed $a<1$

$$
\sum_{n=0}^{\left[\log _{2} m a\right]-1} \max _{2^{n} \leqq t \leqq 2^{n+1}}\left|H_{m}(t)\right|>\sum_{n=0}^{\left[\log _{z} m\right]} \max _{2^{n} \leqq t \leqq 2^{n+1}}\left|H_{m}(t)\right|-A(|\log a|+1) .
$$

Then since $\bar{H}_{m} \uparrow \infty$ as $m \rightarrow \infty$, we can find an $m$ so that $\bar{H}_{m}=H_{m}$ and $m$ also may be chosen so that

$$
\sum_{n=0}^{\left[\log _{2} m a\right]-1} \max _{2^{n} \leqq t \leqq 2^{n+1}}\left|H_{m}(t)\right|>\frac{1}{2} \bar{H}_{m} .
$$

We call the point on $\left(2^{n}, 2^{n+1}\right)$ where $H_{m}(t)$ has maximum absolute value $x_{m n}$. The function $H_{m}(t)$ is continuous and hence there will be an interval $J_{m n}$ containing $x_{m n}$ and contained in $\left(2^{n}, 2^{n+1}\right)$ such that for $x \subset J_{m n}$

$$
\left|H_{m}\left(x_{m n}\right)-H_{m}(x)\right|<\frac{1}{2}\left|H_{m}\left(x_{m n}\right)\right| \text {. }
$$

Then $J_{m n}^{*}$ is defined as the interval into which $J_{m n}$ is carried by the transformation $x^{\prime}=x / m$. It is clear that $J_{m n}^{*}$ is contained in $\left(2^{n} / m, 2^{n+1} / m\right)$. We denote the length of $J_{m n}^{*}$ by $\epsilon_{m n}$. We define

$$
f(x)= \begin{cases}\frac{4 \pi x \operatorname{sgn} H_{m}\left(x_{m n}\right)}{\epsilon_{m n}}, & x \subset J_{m n}^{*}, \quad 0 \leqq n \leqq \log _{2} m a-1, \\ 0, & \text { elsewhere on }\left(\frac{1}{m}, a\right) .\end{cases}
$$

The function $f(x)$ satisfies $(2.9)$, for, if $2^{n} / m \leqq t<2^{n+1} / m\left(0 \leqq n \leqq \log _{2} m a-1\right)$,

$$
\int_{1 / m}^{t}|f(x)| d x \leqq \int_{1 / m}^{2^{n+1 / m}}|f(x)| d x \leqq 4 \pi \sum_{\nu=1}^{n+1} \frac{2^{\nu}}{m}<\frac{4 \pi\left(2^{n+1}-1\right)}{m}<8 \pi t .
$$

By (2.14)

$$
\begin{aligned}
\frac{1}{\pi} \int_{1 / m}^{a} f(x) & \frac{1}{x} H_{m}(m x) d x=\frac{1}{\pi} \sum_{\nu=0}^{\left[\log _{2} m a\right]-1} \int_{J_{m n}^{*}} f(x) \frac{1}{x} H_{m}(m x) d x \\
= & \frac{1}{\pi} \sum_{\nu=0}^{\left[\log _{2} m a\right]-1} \frac{4 \pi \operatorname{sgn} H\left(x_{m n}\right)}{\epsilon_{m n}} \int_{J_{m n}^{*}} H_{m}(m x) d x \\
> & \sum_{n=0}^{\left[\log _{2} m a\right]-1} \frac{4}{\epsilon_{m n}} \epsilon_{m n}\left|\frac{H_{m}\left(x_{m n}\right)}{2}\right|>2 \sum_{n=0}^{\left[\log _{2} m a\right]-1}\left|H_{m}\left(x_{m n}\right)\right|>\bar{H}_{m},
\end{aligned}
$$

so that (2.8) is satisfied and our proof is completed. 
3. $\mathfrak{T}=\tilde{L}$. Fekete $[1]$ has shown that a necessary and sufficient condition that a factor sequence $a_{n}$ carry a series of $\tilde{L}$ into a Fourier series is that

$$
\sum_{n=1}^{\infty} \frac{a_{n}}{n} \cos n t \subset B . V . \text { on }(-\pi, \pi) ;
$$

and

$$
-i \sum_{n=1}^{\infty} a_{n}\left\{f_{n} e^{i n x}-f_{-n} e^{-i n x}\right\} \sim \frac{1}{\pi} \int_{-\pi}^{\pi} f(x+t) d\left\{\sum_{n=1}^{\infty} \frac{a_{n}}{n} \cos n t\right\} .
$$

Therefore

$$
T_{m}(L, x)=\frac{1}{\pi} \int_{-\pi}^{\pi} f(x+t) d\left\{\sum_{n=1}^{\infty} \frac{a_{m n}}{n} \cos n t\right\} .
$$

We make the hypothesis that $a_{m n} \rightarrow 0$ as $n \rightarrow \infty$. This condition is essential here because

$$
\sum_{n=1}^{\infty} \frac{1}{n} \cos n t \notin B . V . \text { on }(-\pi, \pi)
$$

and therefore the argument of $\$ 2$ does not apply. Then

$$
\sum_{n=1}^{\infty} a_{m n} \sin n t=\sum_{n=1}^{\infty} a_{m n}\left\{\tilde{S}_{n}(t)-\tilde{S}_{n-1}(t)\right\}=\sum_{n=1}^{\infty}\left(a_{m n}-a_{m, n+1}\right) \tilde{S}_{n}(t),
$$

where

$$
\tilde{S}_{n}(t)=\sum_{\nu=1}^{n} \sin \nu t=-\frac{1}{2} \cot \frac{t}{2}+\frac{\cos \left(n+\frac{1}{2}\right) t}{2 \sin \frac{1}{2} t} .
$$

Hence, by (1.9),

$$
g(t)=\sum_{n=1}^{\infty} \frac{a_{m n}}{n} \cos n t
$$

is absolutely continuous on every interval not containing the origin and, since $g(-0)=g(+0), g(t)$ can be considered as continuous at the origin so that, since by hypothesis $g(t)$ is of bounded variation, it must be absolutely continuous on $(-\pi, \pi)$. Then

$$
\begin{aligned}
T_{m}(\tilde{L}, x) & =\frac{1}{\pi} \int_{-\pi}^{\pi} f(x+t) \sum_{n=1}^{\infty}\left(a_{m n}-a_{m, n+1}\right) \frac{-\cos \frac{1}{2} t+\cos \left(n+\frac{1}{2}\right) t}{2 \sin \frac{1}{2} t} d t \\
& =\frac{1}{\pi} \int_{0}^{\pi} \phi(\tilde{L}, t) \sum_{n=1}^{\infty}\left(a_{m n}-a_{m, n+1}\right) \frac{-\cos \frac{1}{2} t+\cos \left(n+\frac{1}{2}\right) t}{2 \sin \frac{1}{2} t} d t .
\end{aligned}
$$


By the Riemann-Lebesgue theorem

$$
\int_{0}^{\pi} \phi(\tilde{L}, t)\left\{\frac{\cos \left(n+\frac{1}{2}\right) t}{2 \sin \frac{1}{2} t}-\frac{\cos n t}{t}\right\} d t=o(1) \text { as } n \rightarrow \infty,
$$

so that by the regularity of $\mathscr{A}$

$$
T_{m}(\tilde{L}, x)=\frac{1}{\pi} \int_{0}^{\pi} \phi(\tilde{L}, t)\left\{\frac{-a_{m 1}}{2} \cot \frac{t}{2}+\frac{1}{t} \cdot \tilde{H}_{m}(m t)\right\} d t+o(1) \text { as } m \rightarrow \infty
$$

where

$$
\tilde{H}_{m}(t)=\int_{0}^{\infty} \cos u t d q_{m}(u) .
$$

By the definition of $f(x)$ and the regularity of $\mathfrak{A}$

$$
-\frac{a_{m 1}}{2 \pi} \int_{1 / m}^{\pi} \phi(\tilde{L}, t) \cot \frac{t}{2} d t \rightarrow \tilde{f}(x) \text { as } m \rightarrow \infty,
$$

if $x \subset E(\tilde{L}, f)$, and also,

$$
\int_{0}^{1 / m} \phi(\tilde{L}, t)\left\{\frac{1}{2} \cot \frac{t}{2}-\frac{1}{t}\right\} d t=o(1) \text { as } m \rightarrow \infty,
$$

so that

$$
\begin{aligned}
T_{m}(\tilde{L}, x)-a_{m 1} \tilde{f}(x)= & \frac{1}{\pi} \int_{0}^{1 / m} \phi(\tilde{L}, t) \frac{1}{t}\left\{-a_{m 1}+\tilde{H}_{m}(m t)\right\} d t \\
& +\frac{1}{\pi} \int_{1 / m}^{\pi} \phi(\tilde{L}, t) \frac{1}{t} \tilde{H}_{m}(m t) d t+o(1) \text { as } m \rightarrow \infty .
\end{aligned}
$$

We make a hypothesis analogous to (2.2), namely,

$$
\left|a_{m 1}-\tilde{H}_{m}(t)\right|<B t \text {. }
$$

Then, if $x \subset E(\tilde{L}, f)$,

$$
\begin{aligned}
& \left.\mid \frac{1}{\pi} \int_{0}^{1 / m} \phi \tilde{L}, t\right) \frac{1}{t}\left\{-a_{m 1}+\tilde{H}_{m}(m t)\right\} d t \mid \\
& \quad<\frac{B m}{\pi} \int_{0}^{1 / m}|\phi(\tilde{L}, t)| d t=o(1) \text { as } m \rightarrow \infty .
\end{aligned}
$$

We shall prove that, if $x \subset E(\tilde{L}, f)$,

$$
\tilde{T}_{m}=\frac{1}{\pi} \int_{1 / m}^{\pi} \phi(\tilde{L}, t) \frac{1}{t} \tilde{H}_{m}(m t) d t=o\left(\tilde{H}_{m}\right)+o(1),
$$


where

$$
\tilde{H}_{m}=\sum_{n=0}^{\left[\log _{3} m\right]} \max _{2^{n} \leqq t \leqq 2^{n+1}}\left|\tilde{H}_{m}(t)\right| .
$$

If $a$ is fixed, by the Riemann-Lebesgue theorem and the regularity of $\mathfrak{A}$,

$$
\frac{1}{\pi} \int_{a}^{\pi} \phi(\tilde{L}, t) \frac{1}{t} \tilde{H}_{m}(m t) d t=o(1) \text { as } m \rightarrow \infty,
$$

and

$$
\begin{aligned}
\frac{1}{\pi} \int_{1 / m}^{a} \phi(\tilde{L}, t) \frac{1}{t} \tilde{H}_{m}(m t) d t=\frac{1}{\pi} \int_{1}^{m a} \phi\left(\tilde{L}, \frac{t}{m}\right) \frac{1}{t} \tilde{H}_{m}(t) d t \\
=O\left(\sum_{n=0}^{\left[\log _{2} m\right]} \max _{2^{n} \leqq t \leqq 2^{n+1}}\left|\tilde{H}_{m}(t)\right| 2^{-n} \int_{0}^{2^{n+1}}\left|\phi\left(\tilde{L}, \frac{t}{m}\right)\right| d t\right) \\
=o\left(\tilde{H}_{m}\right) \text { uniformly in } m \text { as } a \rightarrow 0,
\end{aligned}
$$

so that (3.2) must be satisfied.

We would like to prove that this result is the best possible. The method of $\$ 2$ however does not seem to be capable of doing this without some additional assumption. We choose to assume that

$$
\int_{1 / m}^{\pi} \frac{1}{t}\left|\tilde{H}_{m}(m t)\right| d t<K .
$$

This condition is not completely arbitrary for it is analogous to the necessary and sufficient condition for $F$-effectiveness mentioned in $\$ 2$. It is known to be sufficient for $\tilde{F}$-effectiveness but has not been proved to be necessary. It will be seen from the proof that some slightly less restrictive condition such as

$$
\sum_{n=0}^{\left[\log _{2} m\right]} \min _{2^{n} \leqq t \leqq 2^{n+1}}\left|H_{m}(t)\right|<k \tilde{H}_{m}, \quad k<1
$$

could be used.

We define $\tilde{\bar{H}}_{m}=\max _{\mu \leq m} \tilde{H}_{\mu}$ and we wish to show that, if $\tilde{\bar{H}}_{m} \uparrow \infty$ as $m \rightarrow \infty$, and $\left\{d_{m}\right\}$ is any sequence such that $d_{m} \downarrow 0$ as $m \rightarrow \infty$, we can find a function $f(x)$ and a point $x \subset E(\tilde{L}, f)$ for which

$$
\tilde{T}_{m} \neq O\left(d_{m} \tilde{\bar{H}}_{m}\right) \text {. }
$$

As in $\$ 2$ it is sufficient to show that for $a$ fixed it is possible to find a function $f(x)$ and an integer $m$ for which 


$$
\frac{1}{\pi} \int_{1 / m}^{a} f(x) \frac{1}{x} \tilde{H}_{m}(m x) d x>\tilde{H}_{m},
$$

$$
\int_{1 / m}^{t}|f(x)| d x<C t, \quad C \text { not depending on } a \text { or } m,
$$

and

$$
\left|\int_{\epsilon}^{a} \frac{f(x)}{x} d x\right|<D, \quad D \text { not depending on } a \text { or } \epsilon .
$$

For, following the procedure of $\$ 2$, we construct a sequence of functions $f_{i}(x)$ and integers $m_{i}$ such that $m_{0}=1$ and

$$
\begin{gathered}
\sum_{i=1}^{\infty}\left(d_{m_{i}}\right)^{1 / 2}<\infty, \\
\left|\frac{1}{\pi} \int_{1 / m_{j}}^{1 / m_{j-1}} f_{j}(x) \frac{1}{x} \widetilde{H}_{m_{i}}\left(m_{i} x\right) d x\right|<1, \quad j<i, \\
\frac{1}{\pi} \int_{1 / m_{i}}^{1 / m_{i-1}} f_{i}(x) \frac{1}{x} \widetilde{H}_{m_{i}}\left(m_{i} x\right) d x>\tilde{\bar{H}}_{m_{i}}, \\
\int_{1 / m_{i}}^{t}\left|f_{i}(x)\right| d x<C t, \quad \frac{1}{m_{i}} \leqq t \leqq \frac{1}{m_{i-1}},
\end{gathered}
$$

and

$$
\left|\int_{\epsilon}^{1 / m_{i-1}} \frac{f_{i}(x)}{x} d x\right|<D, \quad \frac{1}{m_{i}} \leqq \epsilon \leqq \frac{1}{m_{i-1}},
$$

Then $f(x)$ is defined by

$$
f(x)=\left\{\begin{array}{c}
\left(d_{m_{i}}\right)^{1 / 2} \operatorname{sgn} x f_{i}(|x|),-\frac{1}{m_{i-1}}<x \leqq-\frac{1}{m_{i}}, \frac{1}{m_{i}} \leqq \\
\quad(i=1,2, \cdots) \\
0, \quad-\pi \leqq x \leqq-1, \quad x=0, \quad 1 \leqq x \leqq \pi .
\end{array}\right.
$$

At $x=0, \phi(\tilde{L}, t)=2 f(t)$ and, if $1 / m_{i} \leqq t<1 / m_{i-1}$,

$$
\begin{aligned}
\int_{0}^{t}|\phi(\tilde{L}, t)| d t & =2 \sum_{j=i+1}^{\infty}\left(d_{m_{j}}\right)^{1 / 2} \int_{1 / m_{j}}^{1 / m_{j-1}}\left|f_{j}(x)\right| d x+2\left(d_{m_{i}}\right)^{1 / 2} \int_{1 / m_{i}}^{t}\left|f_{i}(x)\right| d x \\
& <2 C t \sum_{j=i}^{\infty}\left(d_{m_{j}}\right)^{1 / 2}=o(t) \text { as } t \rightarrow 0 .
\end{aligned}
$$

Since $(\cot t / 2-2 / t)$ is a bounded function, the existence of 


$$
\tilde{f}(x)=\lim _{\epsilon \rightarrow 0}\left[-\frac{1}{2 \pi} \int_{\epsilon}^{\pi} \phi(\tilde{L}, t) \cot \frac{t}{2} d t\right]
$$

is equivalent to the existence of

$$
\lim _{t \rightarrow 0}\left[-\frac{1}{\pi} \int_{\epsilon}^{\pi} \phi(\tilde{L}, t) \frac{d t}{t}\right]
$$

By (3.11)

$$
\lim _{\epsilon \rightarrow 0}\left[-\frac{1}{\pi} \int_{\epsilon}^{\pi} \phi(\tilde{L}, t) \frac{d t}{t}\right]=-\sum_{j=1}^{\infty} \frac{2}{\pi}\left(d_{m_{j}}\right)^{1 / 2} \int_{1 / m_{j}}^{1 / m_{j-1}} f_{j}(x) \frac{d x}{x}
$$

and by (3.7) and (3.11) the second sum converges. Therefore the point $x=0$ is contained in $E(\widetilde{L}, f)$. Finally

$$
\begin{aligned}
\tilde{T}_{m_{i}} & =\int_{1 / m_{i}}^{1 / m_{i-1}} \phi(\tilde{L}, t) \tilde{H}_{m_{i}}\left(m_{i} t\right) d t+\int_{1 / m_{i-1}}^{\pi} \phi(\tilde{L}, t) \tilde{H}_{m_{i}}\left(m_{i} t\right) d t \\
& >2\left(d_{m_{i}}\right)^{1 / 2} \tilde{H}_{m_{i}}-O\left(\sum_{j=1}^{i-1}\left(d_{m_{j}}\right)^{1 / 2}\right) \neq O\left(d_{m_{i}} \tilde{H}_{m_{i}}\right) .
\end{aligned}
$$

We now need only construct a function $f(x)$ and an integer $m$ with the properties (3.4), (3.5), and (3.6). First

$$
\left|\tilde{H}_{m}(t)\right|=\left|\int_{0}^{\infty} \cos u t d q_{m}(u)\right|<A,
$$

so that

$$
\sum_{n=0}^{\left[\log _{2} m a\right]-1} \max _{2^{n} \leqq t \leqq 2^{n+1}}\left|\tilde{H}_{m}(t)\right|>\sum_{n=0}^{\left[\log _{2} m\right]} \max _{2^{n} \leqq t \leqq 2^{n+1}}\left|\tilde{H}_{m}(t)\right|-A(|\log a|+1),
$$

and therefore if $\tilde{\bar{H}}_{m} \uparrow \infty$ as $m \rightarrow \infty$, we can find an $m$ so that

$$
\sum_{n=0}^{\left[\log _{2} m a\right]-1} \max _{2^{n} \leqq t \leqq 2^{n+1}}\left|\tilde{H}_{m}(t)\right|-2 K>\frac{1}{2} \tilde{\bar{H}}_{m},
$$

where $K$ is the constant of condition (3.3). We call the point on $\left(2^{n}, 2^{n+1}\right)$ where $\tilde{H}_{m}(t)$ attains its maximum absolute value $x_{m n}$. Since $\tilde{H}_{m}(t)$ is continuous there must be an interval $J_{m n}$ containing $x_{m n}$ and contained in $\left(2^{n}, 2^{n+1}\right)$ such that for $x \subset J_{m n}$

$$
\left|\tilde{H}_{m}\left(x_{m n}\right)-\tilde{H}_{m}(x)\right|<\frac{1}{2} \tilde{H}_{m}\left(x_{m n}\right) .
$$

We call the point on $\left(2^{n}, 2^{n+1}\right)$ where $\tilde{H}_{m}(t)$ attains its minimum absolute value $\bar{x}_{m n}$. Then (3.3) implies that 


$$
\sum_{n=0}^{\left[\log _{2} m\right]}\left|\tilde{H}_{m}\left(\bar{x}_{m n}\right)\right|<K \text {. }
$$

We can find an interval $G_{m n}$ containing $\bar{x}_{m n}$ and contained in $\left(2^{n}, 2^{n+1}\right)$ such that for $x \subset G_{m n}$

$$
\left|\tilde{H}_{m}\left(\bar{x}_{m n}\right)-\tilde{H}_{m}(x)\right|<\frac{1}{8} \tilde{H}_{m}\left(x_{m n}\right) .
$$

We may suppose without loss of generality that the lengths of $J_{m n}$ and $G_{m n}$ are equal. We denote by $J_{m n}^{*}\left(G_{m n}^{*}\right)$ the image of $J_{m n}\left(G_{m n}\right)$ under the transformation $x^{\prime}=x / m$ and we call the length of $J_{m n}^{*}, \epsilon_{m n}$. Then $f(x)$ is defined by

$$
f(x)=\left\{\begin{array}{cl}
\frac{8 \pi x \operatorname{sgn} \tilde{H}_{m}\left(x_{m n}\right)}{\epsilon_{m n}}, & x \in J_{m n}^{*} \\
-\frac{8 \pi x \operatorname{sgn} \tilde{H}_{m}\left(x_{m n}\right)}{\epsilon_{m n}}, & x \subset G_{m n}^{*} \\
0, \quad \text { elsewhere on }\left(\frac{1}{m}, a\right) . & \left(n=0,1, \cdots,\left[\log _{2} m a\right]-1\right),
\end{array}\right.
$$

The function $f(x)$ satisfies (3.4), for

$$
\begin{aligned}
\frac{1}{\pi} \int_{1 / m}^{a} f(x) \frac{1}{x} \tilde{H}_{m}(m x) d x \\
\quad=\frac{1}{\pi} \sum_{n=0}^{\left[\log _{2} m a\right]-1}\left\{\int_{J_{m n}^{*}} f(x) \frac{1}{x} \tilde{H}_{m}(m x) d x+\int_{G_{m n}^{*}} f(x) \frac{1}{x} \tilde{H}_{m}(m x) d x\right\} \\
\quad>4 \sum_{n=0}^{\left[\log _{2} m a\right]-1}\left|\tilde{H}_{m}\left(x_{m n}\right)\right|-8 \sum_{n=0}^{\left[\log _{2} m a\right]-1} \max _{x \in G_{m n}^{*}}\left|\tilde{H}_{m}(m x)\right| \\
>4 \sum_{n=0}^{\left[\log _{2} m a\right]-1}\left|\tilde{H}_{m}\left(x_{m n}\right)\right|-8 \sum_{n=0}^{\left[\log _{2} m a\right]-1}\left|\tilde{H}_{m}\left(\bar{x}_{m n}\right)\right|-8 \sum_{n=0}^{\left[\log _{2} m a\right]-1} \frac{1}{8}\left|\tilde{H}_{m}\left(x_{m n}\right)\right| \\
>2 \tilde{H}_{m}-\tilde{H}_{m}=\tilde{H}_{m} .
\end{aligned}
$$

If $2^{n} / m \leqq t<2^{n+1} / m, 0 \leqq n \leqq\left[\log _{2} m a\right]-1$, we have

$$
\int_{1 / m}^{t}|f(x)| d x \leqq 8 \pi \sum_{\nu=1}^{n+1} \frac{2^{\nu}}{m}<\frac{8 \pi 2^{n+1}}{m}<16 \pi t,
$$

and (3.5) is satisfied. If $2^{n} / m \leqq \epsilon<2^{n+1} / m,\left(n=0,1, \cdots,\left[\log _{2} m a\right]-1\right)$, 


$$
\begin{aligned}
& \int_{\epsilon}^{a} f(x) \frac{d x}{x}=\sum_{\nu=n+1}^{\left[\log _{2} m a\right]-1}\left\{\int_{J_{m n}^{*}} \frac{8 \pi \operatorname{sgn} \tilde{H}_{m}\left(x_{m n}\right)}{\epsilon_{m n}} d x-\int_{G_{m n}^{*}} \frac{8 \pi \operatorname{sgn} \tilde{H}_{m}\left(x_{m n}\right)}{\epsilon_{m n}} d x\right\} \\
& +\int_{2^{n / m}}^{2^{n+1} / m} f(x) \frac{d x}{x} \leqq 8 \pi
\end{aligned}
$$

which proves (3.6) and completes the proof of the theorem.

4. $\mathfrak{T}=L^{\prime}$. In this case we again make the restriction that $a_{m n} \rightarrow 0$ as $n \rightarrow \infty$. This involves no loss of generality for, if $T_{m}\left(L^{\prime}, x\right) \subset L$, then we must have $n a_{m n} f_{n} \rightarrow 0$ as $n \rightarrow \infty$, whenever $f^{\prime}(x)$ is in $L^{\prime}$. This can only be the case if $a_{m n} \rightarrow 0$ as $n \rightarrow \infty$. We have

where

$$
T_{m}\left(L^{\prime}, f\right)=\frac{1}{\pi} \int_{-\pi}^{\pi} d_{t} f(x+t) \sum_{n=0}^{\infty}\left(a_{m n}-a_{m, n+1}\right) \frac{\sin \left(n+\frac{1}{2}\right) t}{2 \sin \frac{1}{2} t},
$$

$$
\sum_{n=0}^{\infty}\left(a_{m n}-a_{m, n+1}\right) \frac{\sin \left(n+\frac{1}{2}\right) t}{2 \sin \frac{1}{2} t} \subset L \text { on }(-\pi, \pi) .
$$

Hence, if $x \subset E\left(L^{\prime}, f\right)$,

$$
T_{m}\left(L^{\prime}, f\right)-a_{m 1} f^{\prime}(x)=\frac{1}{\pi} \int_{0}^{\pi} d \phi_{0}\left(L^{\prime}, t\right) \sum_{n=1}^{\infty}\left(a_{m n}-a_{m, n+1}\right) \frac{\sin \left(n+\frac{1}{2}\right) t}{2 \sin \frac{1}{2} t} .
$$

We wish to determine a necessary and sufficient condition for $L^{\prime}$-effectiveness when the conditions

$$
\begin{gathered}
\left|H_{m}(t)\right|<B t, \\
\sum_{n=0}^{\left[\log _{2} m\right]} \max _{2^{n} \leqq t \leqq 2^{n+1}}\left|H_{m}(t)\right|<M
\end{gathered}
$$

are satisfied. The required condition turns out to be

$$
\sum_{n=0}^{\infty}\left(a_{m n}-a_{m, n+1}\right) \frac{\sin \left(n+\frac{1}{2}\right) t}{2 \sin \frac{1}{2} t} \rightarrow 0 \text { as } m \rightarrow \infty \text { for } t>0 .
$$

This condition is necessary for, if it is not satisfied, let $t_{0}>0$ be a point where

$$
\varlimsup_{m \rightarrow \infty}\left|\sum_{n=0}^{\infty}\left(a_{m n}-a_{m, n+1}\right) \frac{\sin \left(n+\frac{1}{2}\right) t_{0}}{2 \sin \frac{1}{2} t_{0}}\right|>0 .
$$

Then, if $f(x)$ is defined by

$$
f(x)=\left\{\begin{array}{cc}
\operatorname{sgn} x, & t_{0} \leqq|x|<\pi, \\
0, & 0 \leqq|x|<t_{0}, \quad x=\pi
\end{array}\right.
$$

at $x=0, \phi\left(L^{\prime}, t\right)=2 f(t)$ and $f^{\prime}(0)=0$, 


$$
\int_{0}^{t}\left|d \phi_{0}\left(L^{\prime}, \tau\right)\right|=0, \quad t \leqq t_{0},
$$

so that the point $x=0$ is contained in $E\left(L^{\prime}, f\right)$. But

$$
\begin{aligned}
T_{m}\left(L^{\prime}, x\right)-a_{m 1} f^{\prime}(x) & =\frac{1}{\pi} \int_{0}^{\pi} d \phi_{0}\left(L^{\prime}, t\right) \sum_{n=0}^{\infty}\left(a_{m n}-a_{m, n+1}\right) \frac{\sin \left(n+\frac{1}{2}\right) t}{2 \sin \frac{1}{2} t} \\
& =\frac{1}{\pi} \sum_{n=0}^{\infty}\left(a_{m n}-a_{m, n+1}\right) \frac{\sin \left(n+\frac{1}{2}\right) t_{0}}{2 \sin \frac{1}{2} t_{0}}
\end{aligned}
$$

which does not tend to zero as $m \rightarrow \infty$ so the method cannot be $L^{\prime}$-effective.

The condition (4.3) is sufficient, for

$$
\begin{aligned}
T_{m}\left(L^{\prime}, x\right)-a_{m 1} f^{\prime}(x) & =\frac{1}{\pi} \int_{0}^{a} d \phi_{0}\left(L^{\prime}, t\right) \sum_{n=0}^{\infty}\left(a_{m n}-a_{m, n+1}\right) \frac{\sin \left(n+\frac{1}{2}\right) t}{2 \sin \frac{1}{2} t} \\
& +\frac{1}{\pi} \int_{a}^{\pi} d \phi_{0}\left(L^{\prime}, t\right) \sum_{n=0}^{\infty}\left(a_{m n}-a_{m, n+1}\right) \frac{\sin \left(n+\frac{1}{2}\right) t}{2 \sin \frac{1}{2} t} .
\end{aligned}
$$

Since $\sin \left(\left(n+\frac{1}{2}\right) t\right) /\left(2 \sin \left(\frac{1}{2} t\right)\right)-(\sin n t) / t$ is bounded, if $x \subset E\left(L^{\prime}, f\right)$,

$$
\begin{aligned}
\frac{1}{\pi} \int_{0}^{a} d \phi_{0}\left(L^{\prime}, t\right) & \sum_{n=0}^{\infty}\left(a_{m n}-a_{m, n+1}\right) \frac{\sin \left(n+\frac{1}{2}\right) t}{2 \sin \frac{1}{2} t} \\
= & \frac{1}{\pi} \int_{0}^{a} d \phi_{0}\left(L^{\prime}, t\right) \frac{1}{t} H_{m}(m t)+o(1) \text { uniformly in } m \text { as } a \rightarrow 0,
\end{aligned}
$$

and, by (4.1) and (4.2),

$$
\begin{aligned}
& \frac{1}{\pi} \int_{0}^{1 / m} d \phi_{0}\left(L^{\prime}, t\right) \frac{1}{t} H_{m}(m t)<\frac{B m}{\pi} \int_{0}^{1 / m}\left|d \phi_{0}\left(L^{\prime}, t\right)\right|=o(1) \text { as } m \rightarrow \infty, \\
& \begin{aligned}
\frac{1}{\pi} \int_{1 / m}^{a} d \phi_{0}\left(L^{\prime}, t\right) & \frac{1}{t} H_{m}(m t) \\
& =O\left(\sum_{n=0}^{\left[\log _{2} m a\right]} \max _{2^{n} \leqq t 2^{n+1}}\left|H_{m}(t)\right| 2^{-n} m \int_{0}^{2^{n+1}}\left|d \phi_{0}\left(L^{\prime}, t / m\right)\right|\right) \\
& =o(1) \text { uniformly in } m \text { as } a \rightarrow 0 .
\end{aligned}
\end{aligned}
$$

Finally, if (4.3) is satisfied, since

$$
\begin{gathered}
\sum_{n=1}^{\infty}\left(a_{m n}-a_{m, n+1}\right) \frac{\sin \left(n+\frac{1}{2}\right) t}{2 \sin \frac{1}{2} t}=o\left(\frac{A}{t}\right), \\
\frac{1}{\pi} \int_{a}^{\pi} d \phi_{0}\left(L^{\prime}, t\right) \sum_{n=0}^{\infty}\left(a_{m n}-a_{m, n+1}\right) \frac{\sin \left(n+\frac{1}{2}\right) t}{2 \sin \frac{1}{2} t}=o(1) \text { as } m \rightarrow \infty .
\end{gathered}
$$

By putting these results together we have the proof of sufficiency. 
5. $\mathfrak{T}=\tilde{L}^{\prime}$. In this case as in the case considered in $\$ 4$ the condition $a_{m n} \rightarrow 0$ as $n \rightarrow \infty$ is essential in order that $T_{m}\left(\tilde{L}^{\prime}, x\right) \subset L$. Then

$$
\begin{aligned}
T_{m}\left(\tilde{L}^{\prime}, x\right) & =\frac{1}{\pi} \int_{-\pi}^{\pi} d_{t} f(x+t) \sum_{n=1}^{\infty} a_{m n} \sin n t \\
& =\frac{1}{\pi} \int_{-\pi}^{\pi} d_{t} f(x+t) \sum_{n=1}^{\infty}\left(a_{m n}-a_{m, n+1}\right) \frac{-\cos \frac{1}{2} t+\cos \left(n+\frac{1}{2}\right) t}{2 \sin \frac{1}{2} t} \\
& =\frac{1}{\pi} \int_{0}^{\pi} d \phi_{0}\left(\tilde{L}^{\prime}, t\right) \sum_{n=1}^{\infty}\left(a_{m n}-a_{m, n+1}\right) \frac{-\cos \frac{1}{2} t+\cos \left(n+\frac{1}{2}\right) t}{2 \sin \frac{1}{2} t} .
\end{aligned}
$$

We shall assume that

$$
\left|a_{m 1}-\widetilde{H}_{m}(t)\right|<B t
$$

and

$$
\sum_{n=1}^{\left[\log _{2} m\right]} \max _{2^{n} \leqq t 2^{n+1}}\left|\tilde{H}_{m}(t)\right|<M,
$$

and we propose to find a necessary and sufficient condition for $\tilde{L}^{\prime}$-effectiveness. This condition is

$$
\sum_{n=1}^{\infty}\left(a_{m n}-a_{m, n+1}\right) \frac{\cos \left(n+\frac{1}{2}\right) t}{\sin \frac{1}{2} t} \rightarrow 0 \text { as } m \rightarrow \infty, \text { for } t>0 .
$$

The condition is necessary for, if it is not satisfied, let $t_{0}>0$ be a point such that

$$
\varlimsup_{m \rightarrow \infty}\left|\sum_{n=1}^{\infty}\left(a_{m n}-a_{m, n+1}\right) \frac{\cos \left(n+\frac{1}{2}\right) t_{0}}{\sin \frac{1}{2} t_{0}}\right|>0 .
$$

Then we define

$$
f(x)= \begin{cases}1, & -\pi \leqq x \leqq-t_{0}, \quad t_{0} \leqq x \leqq \pi \\ 0, & -t_{0}<x<t_{0} .\end{cases}
$$

For this function at $x=0, \phi_{0}\left(\tilde{L}^{\prime}, t\right)=2 f(t)$ and

$$
\tilde{f}^{\prime}(0)=\frac{-1}{2 \pi} \int_{0}^{\pi} d \phi_{0}\left(\tilde{L}^{\prime}, t\right) \cot \frac{t}{2}=-\frac{1}{\pi} \cot \frac{t_{0}}{2} .
$$

Also

$$
\int_{0}^{t}\left|d \phi_{0}\left(\mathcal{L}^{\prime}, t\right)\right|=0, \text { for } t<t_{0}
$$


so that the point $x=0$ is contained in $E\left(\tilde{L}^{\prime}, f\right)$. But

$$
\begin{aligned}
T_{m}\left(\tilde{L}^{\prime}, x\right) & =\frac{1}{\pi} \int_{0}^{\pi} d \phi_{0}\left(\tilde{L}^{\prime}, t\right) \sum_{n=1}^{\infty}\left(a_{m n}-a_{m, n+1}\right) \frac{-\cos \frac{1}{2} t+\cos \left(n+\frac{1}{2}\right) t}{2 \sin \frac{1}{2} t} \\
& =a_{m 1} \tilde{f}^{\prime}(0)+\frac{1}{\pi} \sum_{n=1}^{\infty}\left(a_{m n}-a_{m, n+1}\right) \frac{\cos \left(n+\frac{1}{2}\right) t_{0}}{\sin \frac{1}{2} t_{0}}
\end{aligned}
$$

which cannot tend to $\tilde{f}^{\prime}(0)$ as $m \rightarrow \infty$ and hence the method is not $\tilde{L}^{\prime}$-effective. If (5.3) is satisfied and $x \subset E\left(\tilde{L}^{\prime}, f\right)$,

$$
\begin{aligned}
& \frac{1}{\pi} \int_{a}^{\pi} d \phi_{0}\left(\tilde{L}^{\prime}, t\right) \sum_{n=1}^{\infty}\left(a_{m n}-a_{m, n+1}\right) \frac{\cos \left(n+\frac{1}{2}\right) t}{2 \sin \frac{1}{2} t} \rightarrow 0 \text { as } m \rightarrow \infty, \\
& \quad-\frac{1}{2 \pi} \int_{1 / m}^{\pi} d \phi_{0}\left(\tilde{L}^{\prime}, t\right) a_{m 1} \cot \frac{t}{2} \rightarrow \tilde{f}^{\prime}(x) \text { as } m \rightarrow \infty, \\
& \frac{1}{\pi} \int_{0}^{1 / m} d \phi_{0}\left(\tilde{L}^{\prime}, t\right) \sum_{n=1}^{\infty}\left(a_{m n}-a_{m, n+1}\right) \frac{-\cos \frac{1}{2} t+\cos \left(n+\frac{1}{2}\right) t}{2 \sin \frac{1}{2} t} \\
& =\frac{1}{\pi} \int_{0}^{1 / m} d \phi_{0}\left(\tilde{L}^{\prime}, t\right) \sum_{n=1}^{\infty}\left(a_{m n}-a_{m, n+1}\right) \frac{-1+\cos n t}{t}+O\left(\int_{0}^{1 / m}\left|d \phi_{0}\left(\tilde{L}^{\prime}, t\right)\right|\right) \\
& =O\left(\frac{m}{\pi} \int_{0}^{1 / m}\left|d \phi_{0}\left(\tilde{L}^{\prime}, t\right)\right|\right)+o(1)=o(1) \text { as } m \rightarrow \infty,
\end{aligned}
$$

and

$$
\begin{aligned}
\frac{1}{\pi} \int_{1 / m}^{a} d \phi_{0} & \left(\tilde{L}^{\prime}, t\right) \sum_{n=1}^{\infty}\left(a_{m n}-a_{m, n+1}\right) \frac{\cos \left(n+\frac{1}{2}\right) t}{2 \sin \frac{1}{2} t} \\
& =\frac{1}{\pi} \int_{1 / m}^{a} d \phi_{0}\left(\tilde{L}^{\prime}, t\right) \sum_{n=1}^{\infty}\left(a_{m n}-a_{m, n+1}\right) \frac{\cos n t}{t}+o(1) \\
& =O\left(\sum_{n=1}^{\left[l \log _{2} m a\right]} \max _{2^{n} \leqq t \leqq 2^{n+1}}\left|\tilde{H}_{m}(t)\right| 2^{-n} m \int_{0}^{2^{n+1}}\left|d \phi_{0}\left(\tilde{L}^{\prime}, t / m\right)\right|\right)+o(1) \\
& =o(1) \text { uniformly in } m \text { as } a \rightarrow \infty .
\end{aligned}
$$

Therefore conditions (5.1), (5.2), and (5.3) are sufficient for $\tilde{L}^{\prime}$-effectiveness.

6. A necessary condition. We shall consider methods of summability for which

$$
\int_{N}^{\infty}\left|d q_{m}(u)\right| \rightarrow 0 \text { uniformly in } m \text { as } N \rightarrow \infty
$$

and 


$$
q_{m}(u) \rightarrow q(u) \text { as } m \rightarrow \infty \text {, over an everywhere dense set. }
$$

The definition of $q(u)$ could be extended to the whole interval $(0, \infty)$ by setting

$$
q(u)=\frac{1}{2}\{q(u+0)+q(u-0)\}
$$

and $q(u)$ would have the property

$$
\int_{0}^{\infty}|d q(u)|<A .
$$

Under these assumptions we have

$$
H_{m}(t) \rightarrow H(t)=\int_{0}^{\infty} \sin u t d q(u)
$$

for

$$
\left|H_{m}(t)-H(t)\right| \leqq\left|\int_{0}^{N} \sin u t d\left\{q_{m}(u)-q(u)\right\}\right|+\int_{N}^{\infty}\left|d q_{m}(u)\right|+\int_{N}^{\infty}|d q(u)|
$$

and, by (6.1),

$$
\int_{N}^{\infty}|d q(u)|+\int_{N}^{\infty}\left|d q_{m}(u)\right| \rightarrow 0 \text { uniformly in } m \text { as } N \rightarrow \infty,
$$

and for $N$ fixed

$$
\begin{aligned}
\mid \int_{0}^{N} \sin u t d & \left\{q_{m}(u)-q(u)\right\} \mid \\
& \leqq t \int_{0}^{N}\left|q_{m}(u)-q(u)\right| d u+\left|q_{m}(N-0)-q(N-0)\right| \\
& =o(1) \text { as } m \rightarrow \infty .
\end{aligned}
$$

Combining these results we see that $H_{m}(t) \rightarrow H(t)$ as $m \rightarrow \infty$.

We propose to show that a necessary condition for $L$-effectiveness, if (2.2) is satisfied, is

$$
\sum_{n=0}^{\infty} \max _{2^{n} \leqq t 2^{n+1}}|H(t)|<M .
$$

If (6.3) is not satisfied then for every $M$, we can find an $N$ such that

$$
\sum_{n=0}^{N}\left|H\left(t_{n}\right)\right|>M,
$$


where $t_{n}$ is a point on $\left(2^{n}, 2^{n+1}\right)$ where $H(t)$ attains its maximum absolute value. We choose $m>2^{N}$ so that

$$
\left|H_{m}\left(t_{n}\right)-H\left(t_{n}\right)\right|<\frac{M}{2 N}, \quad 0 \leqq n \leqq N .
$$

Then

$$
\sum_{n=0}^{\left[\log _{2} m\right]} \max _{2^{n} \leqq t \leqq 2^{n+1}}\left|H_{m}(t)\right|>\sum_{n=0}^{N}\left|H_{m}\left(t_{n}\right)\right|-\frac{M}{2}>\frac{M}{2},
$$

and hence $H_{m}$ cannot be bounded. Therefore condition (6.3) is necessary for $L$-effectiveness.

After the same fashion it can be shown that, if (6.1) and (6.2) are satisfied,

$$
\widetilde{H}_{m}(t) \rightarrow \widetilde{H}(t)=\int_{0}^{\infty} \cos u t d q(u) \text { as } m \rightarrow \infty,
$$

and, if (3.1) and (3.3) are satisfied, then a necessary condition for $\tilde{L}$-effectiveness is

$$
\sum_{n=0}^{\infty} \max _{2^{n} \leqq t \leqq 2^{n+1}}|\tilde{H}(t)|<\infty .
$$

7. Methods of the closed cycle. As an application of these results we shall consider the problem of methods of the closed cycle. For such methods $a_{m n}=a(n / m)$. The function $a(x)$ may be considered to be defined everywhere on $(0, \infty)$. Then (1.9) implies that

$$
\int_{0}^{\infty}|d a(x)|<A
$$

and we have, at every point of continuity of $a(u)$,

$$
q_{m}(u)=a\left(\frac{[u m]}{m}\right)-a(0) \rightarrow a(u)-a(0) \text { as } m \rightarrow \infty .
$$

so

$$
\int_{N}^{\infty}\left|d q_{m}(u)\right| \leqq \int_{N}^{\infty}|d a(u)| \rightarrow 0 \text { as } N \rightarrow \infty,
$$

so that the conditions of $\S 6$ are satisfied. Therefore, if (2.2) is satisfied a necessary condition for $L$-effectiveness is

$$
\sum_{n=0}^{\infty} \max _{2^{n} \leqq t \leqq 2^{n+1}}|H(t)|<\infty,
$$


where

$$
H(t)=\int_{0}^{\infty} \sin u t d a(u) .
$$

We propose to show that this condition is also sufficient. Since $H(t)$ is bounded and, by (2.2), $|H(t)|<B t$, the function $(1 / t) H(t) \subset L_{2}$ on $(0, \infty)$. Therefore it will have a Fourier transform contained in $L_{2}$, and since, if $a(x) \rightarrow 0$ as $x \rightarrow \infty$,

$$
\frac{1}{t} H(t)=\int_{0}^{\infty} \cos u t a(u) d u
$$

$(2 / \pi)^{1 / 2} a(x)$ will be this transform. Condition (7.1) implies that $(1 / t) H(t) \subset L$ and therefore

$$
a(x)=\frac{2}{\pi} \int_{0}^{\infty} \cos x t \frac{1}{t} H(t) d t
$$

By the Fubini theorem, if $H(-t)=H(t)$, the function

$$
\frac{1}{\pi} \int_{-\infty}^{\infty} f(x+t) \frac{1}{t} H(m t) d t
$$

will be integrable on $(-\pi, \pi)$, since (7.1) implies that $(1 / t) H(t) \subset L$ on $(0, \infty)$. Let us now consider its Fourier series. We have

$$
\begin{aligned}
\frac{1}{2 \pi} \int_{-\pi}^{\pi} e^{-i n x} & \frac{1}{\pi} \int_{-\infty}^{\infty} f(x+t) \frac{1}{t} H(m t) d t d x \\
= & \frac{1}{\pi} \int_{-\infty}^{\infty} \frac{1}{t} H(m t) \frac{1}{2 \pi} \int_{-\pi}^{\pi} f(x+t) e^{-i n x} d x d t \\
& =\frac{1}{\pi} \int_{-\infty}^{\infty} \frac{1}{t} H(m t) f_{n} e^{i n t} d t=f_{n} a(|n| / m)
\end{aligned}
$$

Therefore

$$
\frac{1}{\pi} \int_{-\infty}^{\infty} f(x+t) \frac{1}{t} H(m t) d t \sim \sum_{n=-\infty}^{\infty} a(|n| / m)\left\{f_{n} e^{i n x}+f_{n} e^{-i n x}\right\},
$$

or

$$
T_{m}(L, x)=\frac{1}{\pi} \int_{-\infty}^{\infty} f(x+t) \frac{1}{t} H(m t) d t=\frac{1}{\pi} \int_{0}^{\infty} \phi(L, t) \frac{1}{t} H(m t) d t .
$$

Now, if $x \subset E(L, f)$ and (2.2), (7.1) are satisfied, 


$$
\left|\frac{1}{\pi} \int_{0}^{1 / m} \phi(L, t) \frac{1}{t} H(m t) d t\right|<\frac{B m}{\pi} \int_{0}^{1 / m}|\phi(L, t)| d t=o(1) \text { as } m \rightarrow \infty,
$$

and

$$
\begin{aligned}
& \left|\int_{a}^{\infty} \phi(L, t) \frac{1}{t} H(m t) d t\right|=\left|\int_{m a}^{\infty} \phi\left(L, \frac{t}{m}\right) \frac{1}{t} H(t) d t\right| \\
& \quad \leqq \sum_{n=\left[\log _{2} m a\right]}^{\infty} \max _{2^{n} \leqq t \leqq 2^{n+1}}|H(t)| 2^{-n} \int_{0}^{2^{n+1}}\left|\phi\left(L, \frac{t}{m}\right)\right| d t \\
& \quad=O\left(\sum_{n=\left[\log _{2} m a\right]}^{\infty} \max _{2^{n} \leqq t \leqq 2^{n+1}}|H(t)|\right)=o(1) \text { as } m \rightarrow \infty .
\end{aligned}
$$

Finally

$$
\begin{aligned}
& \left|\int_{1 / m}^{a} \phi(L, t) \frac{1}{t} H(m t) d t\right|=\left|\int_{1}^{m a} \phi\left(L, \frac{t}{m}\right) \frac{1}{t} H(t) d t\right| \\
& \quad \leqq \sum_{n=0}^{\left[\log _{2} m a\right]} \max _{2^{n} \leqq t \leqq 2^{n+1}}|H(t)| 2^{-n} \int_{0}^{2^{n+1}}\left|\phi\left(L, \frac{t}{m}\right)\right| d t \\
& \quad=o\left(\sum_{n=0}^{\infty} \max _{2^{n} \leqq t \leqq 2^{n+1}}|H(t)|\right)=o(1) \text { uniformly in } m \text { as } a \rightarrow 0 .
\end{aligned}
$$

From this it follows that (7.1) is necessary and sufficient for $L$-effectiveness, if (2.2) is satisfied. This result was conjectured by Paley in an unpublished paper but was not proved.

Similarly, if

$$
\widetilde{H}(t)=\int_{0}^{\infty} \cos u t d a(u)
$$

it can be shown that

$$
T_{m}(\tilde{L}, x)=\frac{1}{\pi} \int_{0}^{\infty} \phi(\mathcal{L}, t) \frac{1}{t}(-1+\tilde{H}(m t)) d t,
$$

and, if (3.1) and (3.3) are satisfied, a necessary and sufficient condition for $\tilde{L}$-effectiveness is

$$
\sum_{n=0}^{\infty} \max _{2^{n} \leqq t \leqq 2^{n+1}}|\tilde{H}(t)|<\infty .
$$

8. Discussion. From the preceding work there would seem to be apparent differences between $F$-effectiveness, $L$-effectiveness, and $L^{\prime}$-effectiveness and also between $\tilde{F}$-effectiveness, $\tilde{L}$-effectiveness, and $\tilde{L}^{\prime}$-effectiveness. Examples 
have been given (see Paley, Randels, and Rosskopf [4] and Randels [5, Parts I and II]) showing that these differences are essential. It would be of interest to find relations between effectiveness for Fourier series and for conjugate series. One trivial result in this direction is possible, for we have seen that the $L$-effectiveness problem is not changed if $a_{m}=\lim _{n \rightarrow \infty} a_{m n} \neq 0$ while our discussion of $\tilde{L}$-effectiveness is only valid if $a_{m}=0$. It is clear then that it is possible to construct methods of summability which are $L$-effective but not $\tilde{L}$-effective. It would be of much more interest to find out if this is still possible when $a_{m}=0$.

It is natural to ask whether it would be possible to dispense with the conditions (2.2) and (3.1) and still get necessary and sufficient conditions. It appears to be quite definite that this method cannot accomplish this.

\section{REFERENCES}

1. M.Fekete, Über Faktorenfolgen welche die "Klasse" einer Fourierschen Reihe unverändert lassen, Acta Szeged, Universität Franz Joseph, vol. 1 (1923), pp. 148-166.

2. E. Hille and J. D. Tamarkin, On the summability of Fourier series, I, these Transactions, vol. 34 (1932), pp. 757-783.

3. E. Hille and J. D. Tamarkin, On the summability of Fourier series, fifth note, Proceedings of the National Academy of Sciences, vol. 20 (1934), pp. 369-372.

4. R. E. A. C. Paley, W. C. Randels, and M. F. Rosskopf, On the summation of Fourier series, Bulletin of the American Mathematical Society, vol. 40 (1934), pp. 69-74.

5. W. C. Randels, Three examples in the theory of Fourier series, Annals of Mathematics, (2), vol. 36 (1935), pp. 838-858.

INSTITUTE FOR ADVANCED STUDY,

Princeton, N. J. 